\title{
Properties of Extrusion Processed Corn and Corn Coproducts
}

\author{
Jordan J. Rich ${ }^{1} \&$ Kurt A. Rosentrater ${ }^{1}$ \\ ${ }^{1}$ Department of Agricultural and Biosystems Engineering, Iowa State University, 3327 Elings Hall, Ames, IA \\ 50011, USA \\ Correspondence: Kurt A. Rosentrater, Department of Agricultural and Biosystems Engineering, Iowa State \\ University, 3327 Elings Hall, Ames, IA 50011, USA. Email: karosent@ iastate.edu
}

Received: July 23, 2016

Accepted: September 7, 2016 Online Published: October 13, 2016

doi:10.5539/jfr.v5n6p21

URL: http://dx.doi.org/10.5539/jfr.v5n6p21

\begin{abstract}
As the world population continues to grow, the demand for human food and animal feed grows exponentially. Aquaculture is the food sector which has been growing at the greatest rate for several years. Because of the expense of fishmeal in aquaculture fees, an inexpensive protein source could be corn-based proteins. Although many studies have focused on the effects of extruding corn-based blends along with other supplement ingredients, few studies have focused on the extrusion of individual corn-based ingredients. This study examined physical effects of extrusion on distillers dried grains with soluble (DDGS) and corn. Specific objectives included determining moisture content, water activity, color, unit density, durability, water stability, floatability, and bulk density for each corn-based extrudate. Blends were prepared with three levels of moisture $(15,25$, and $35 \% \mathrm{db}$ ), and extrusion conditions included three screw speeds $(50,75$, and $100 \mathrm{rpm})$ and three barrel temperatures $\left(100,125\right.$, and $\left.150^{\circ} \mathrm{C}\right)$. Results showed that as the moisture content increased, the water activity increased in the raw ingredients, and the moisture content of the extrudates increased. As the screw speed increased, the bulk density decreased in the extrudates, and the mass flow rate increased. As the temperature increased, the floatability of the extrudates increased, while the bulk density decreased. The amount of protein and starch content in the corn products affected the physical quality of the pellets, which is important in aquaculture feed development.
\end{abstract}

Keywords: extrusion, corn, DDGS, aquaculture, feed

\section{Introduction}

As the world's population continues to grow exponentially, the need for efficient food and feed production increases each day. Snack foods and breakfast cereals are among many food industries that have grown in the United States, and many of these are produced through extrusion (Razzaq et al., 2012).

Along with the human food industry, aquaculture is one of the fastest growing food production activities globally. It plays a major role in many third world countries because it produces relatively higher income, better nutrition, and better employment opportunities (Kannadhason et al., 2008). Nutritional feed for aquaculture is costly, and the feed cost is generally 30\% to 60\% of total operational costs (Kannadhason et al., 2009, 2010), both due to raw ingredient costs as well as processing costs. Because feed is so expensive, it is difficult to provide the fish appropriate nutrients they need to thrive.

Extrusion has become a popular processing technique in the feed, cereal, and snack food industries (Delgado-Nieblas et al., 2012). Extrusion is one of the most versatile unit operations (Forsido and Ramaswamy, 2011). Extrusion systems consist of barrel housing with one or two rotating screws, a preconditioner, and an accompanying machine control system (Sorensen, 2012). Extrusion cooking uses high temperatures in a relatively short time to move the ingredients through the barrel and exit through the die. The goals of the extrusion process include cooking, sterilization, expansion, texturization, and product shaping. These factors are especially important in aquaculture feeds because porous pellets that float in water are required. Moreover, these are all important factors for human foods as well (Liu and Rosentrater, 2011).

During the extrusion process, starch is gelatinized, which plays an important role in the final extrudate properties (Chevanan et al., 2008a). Gelatinization is crucial because it affects feed digestibility, expansion, and contributes to water solubility and particle binding (Rosentrater et al., 2009a,b). Along with starch gelatinization, protein denaturation and destruction of microbes and other toxic compounds are other reactions that may also occur 
during cooking (Kannadhason et al., 2008). The sterilization process allows for the termination of pathogens, which is important in food products for humans, as well as fish (Liu and Rosentrater, 2011).

In the Midwest U.S., corn and soybean crops are the most prevalent for food production and processing. Both corn and soy are relatively high in protein, which is important for aquaculture feeds. According to a study performed at North Dakota State University, corn contains approximately 55-60\% escape or bypass protein (Lardy, 2002). The United Soybean Board found soybeans contain 35-38\% protein (The United Soybean Board [USB], 2012). Many types of concentrated and isolated meals have been produced for livestock feed. Using the high-protein feed produced for livestock has potential to be extruded for use in aquaculture feed.

Changes of the extrusion conditions, especially barrel temperature, screw speed, and moisture content can have significant effects on the resulting physical properties of the extrudates (Singh et al., 2012). Physical properties are important because they impact the quality of the extrudates. Moisture content is a determining factor for cohesiveness (Ayadi et al., 2011a). Water activity values indicate shelf life (Ayadi et al., 2011b). Because spoilage microorganisms thrive in water activity levels greater than 0.5 , a value less than 0.5 is preferred. The greater the values of water activity, the more likely the microorganisms are to thrive, so a lower water activity value is preferred. Simons found that as extruder screw speed increases, water activity decreases (Simons et al., 2012). Color of the extrudate indicates, to some extent, the nutritional quality of the product (Kannadhason et al., 2010), as darker colors can indicate protein degradation. The mass flow rate quantifies the processing performance of the extruder (Ayadi et al., 2011c). Rosentrater found that an increase in both moisture content and temperature result in a decrease in die pressure (Rosentrater et al., 2009a,b). Chevanan found that increasing the moisture content resulted in an increase in pellet durability (Chevanan et al., 2007a). They also found that increasing the temperature resulted in a decrease in apparent viscosity and pellet durability. Chevanan also found that changes in screw speed had a significant effect on all extrusion parameters except for the temperature of the die (Chevanan et al., 2008b). They also found that increasing the screw speed resulted in a decrease in bulk density because of greater expansion (Chevanan et al., 2007b).

The ingredients used in feed extrusion must generally be high in protein, because aquaculture feeds typically required 26 to 50\% protein, depending on the fish type, species, and age (Chevanan et al., 2007c). Because of the recent biofuel revolution, the production of ethanol has grown immensely across the United States. A coproduct of ethanol manufacturing is distillers dried grains with solubles (DDGS), which contains moderate levels of protein, fat, and fairly high levels of fiber. Because of the nutritional value provided by DDGS, it has become a key ingredient in feeds for many species, including ruminants and non-ruminants. Research on using DDGS in fish feeds is also promising (Liu and Rosentrater, 2011).

The purpose of this study was to evaluate the effects of extrusion conditions on DDGS, focusing on the effects of ingredient moisture content, extruder screw speed, and barrel temperature. In order to find optimal extrudate properties, extensive physical property testing was conducted after the extrusion process, and results were compared to corn - based extrudate properties.

\section{Materials and Methods}

\subsection{Sample Preparation}

Corn ingredients, including DDGS and corn (for comparison purposes), were ground to $<1.0 \mathrm{~mm}$ particle size using a laboratory mill (Model 4, THOMAS-Wiley, Swedesboro, NJ, USA). The ingredients were then adjusted to three target moisture contents $(15,25$, and $35 \% \mathrm{db})$ by adding appropriate amounts of water. The ingredients and water were mixed for approximately $20 \mathrm{~min}$, using a small rotary mixer (Model 043206, Type B, Kobalt, Surrey, BC, Canada). The water was added slowly using a spray bottle to prevent agglomeration. The blends were stored overnight to allow for the distribution and equilibration of moisture at room temperature $\left(25 \pm 1^{\circ} \mathrm{C}\right)$.

\subsection{Extrusion Processing}

A single-screw extruder (Model PL2000, Type 680143, C. W. Brabender, South Hackensack, NJ, USA) was used for the cooking process. The screw compression ratio was $1: 1$, and the diameter of the die was $3.05 \mathrm{~mm}$. The temperature of the feeding zone was room temperature $\left(25 \pm 1^{\circ} \mathrm{C}\right)$, the metering zone was adjusted to $75^{\circ} \mathrm{C}$, and the die zone was adjusted to one of three temperatures $\left(100,125\right.$, and $\left.150^{\circ} \mathrm{C}\right)$ throughout the process. The extruder was capable of a screw speed ranging from 0-100 rpm, so the screw speed was adjusted to three speeds $(50,75$, and $100 \mathrm{rpm})$ for the appropriate treatments. Once the processing was completed, the extrudates were stored for approximately $72 \mathrm{~h}$ at room temperature $\left(25 \pm 1^{\circ} \mathrm{C}\right)$, allowing them to air dry.

\subsection{Measurement of Extrudate Properties}

The extrudates were subjected to extensive physical property analyses, including moisture content $(\% \mathrm{db})$, water 
activity $\left(\mathrm{a}_{\mathrm{w}},-\right)$, color $\left(\mathrm{L}^{*}, \mathrm{a}^{*}, \mathrm{~b}^{*}\right)$, unit density $\left(\mathrm{g} / \mathrm{cm}^{3}\right)$, pellet durability $(\%)$, water stability (s), floatability (s), and bulk density $\left(\mathrm{kg} / \mathrm{m}^{3}\right)$. The mass flow rate $(\mathrm{g} / \mathrm{min})$ and the temperature of the feeding, metering, and die zones $\left({ }^{\circ} \mathrm{C}\right)$ were recorded during the extrusion process.

\subsubsection{Moisture Content (MC)}

Moisture content was determined using approved American Association of Cereal Chemists [AACC] method 44-19 (2000) with a laboratory oven (HERAtherm, Thermo Scientific, Waltham, MA, USA). The samples were prepared and heated for $2 \mathrm{~h}$ at $135^{\circ} \mathrm{C}$. Moisture content was determined for both the raw ingredients, as well as the extrudates.

\subsubsection{Water Activity $\left(\mathrm{a}_{\mathrm{w}}\right)$}

The water activity was determined for both the raw ingredients, as well as the extrudates using a water activity meter (Series 3TE, AquaLab, Pullman, WA, USA). The meter was calibrated and a sample cup was filled with each sample and placed inside the reading chamber.

\subsubsection{Color}

Color $\left(\mathrm{L}^{*}, \mathrm{a}^{*}\right.$, and $\left.\mathrm{b}^{*}\right)$ of the raw ingredients and extrudates was determined using a spectrophotometer (LabScan XE, HunterLab, Reston, VA, USA). $\mathrm{L}^{*}$ is the brightness/darkness, $\mathrm{a}^{*}$ is the redness/greenness, and $\mathrm{b}^{*}$ is the yellowness/blueness.

\subsubsection{Unit Density (UD)}

Extrudates were cut using a razor blade into pieces approximately $2 \mathrm{~cm}$ long. Each extrudate was weighed on an electric balance (Model AV114, OHAUS Adventurer Pro, Pine Brook, NJ, USA), and the length and diameter were determined using a digital calliper (Model 14-648-17, Fisher Scientific, Pittsburgh, PA, USA). The unit density was defined as the ratio between the mass compared to the volume, which was based on the cylindrical shape of the extrudate.

\subsubsection{Pellet Durability Index (PDI)}

Approximately $200 \mathrm{~g}$ of extrudates were broken into pieces approximately $2 \mathrm{~cm}$ long, then placed in a pellet durability tester (Model PDT-110, Gamet Automatic Sampling Equipment, St. Paul, MN, USA) for a 10-min tumbling period. After tumbling, the samples were then sieved using a no. 8 sieve (2.36 mm) (Model 122622665, Fisher Scientific, Pittsburgh, PA, USA). The pellet durability index was calculated using the following equation:

$$
\text { PDI }=\left(\mathrm{M}_{\mathrm{at}} / \mathrm{M}_{\mathrm{bt}}\right) \times 100
$$

Where,

PDI $=$ Pellet durability index $(\%)$

$\mathrm{M}_{\mathrm{at}}=$ Mass of the pellets after tumbling $(\mathrm{g})$

$\mathrm{M}_{\mathrm{bt}}=$ Mass of the pellets before tumbling $(\mathrm{g})$

Bulk Density (BD): Using the method described by the USDA (1999), bulk density was measured using a standard bushel tester (Model 151, Seedburo Equipment Co, Chicago, IL, USA) and a test weight 1-L cup and leveling stick (Model 103, Seedburo Equipment Co, Chicago, IL, USA).

\subsubsection{Mass Flow Rate}

The mass flow rate was determined by collecting extrudates at the die for 30-s intervals, and then weighing them on an electronic balance (Model EX10201, Ohaus Explorer, Pine Brook, NJ, USA). The extrudates were then dried to determine the moisture content, which gave the dry-basis mass flow rate.

\subsubsection{Statistical Analysis}

Raw ingredients were adjusted to three moisture content levels $(15,25$, and $35 \% \mathrm{db})$. The blends were extruded at three die temperatures $\left(100,125\right.$, and $\left.150^{\circ} \mathrm{C}\right)$ and three screw speeds $(50,75$, and $100 \mathrm{rpm})$. Each of the factors included a high point and a low point with a central composite point, which resulted in 9 treatments (i.e. $2 \times 2 \times 2=8$, plus 1 center point). The experimental protocol is shown in Table 1 . Most of the physical properties were measured in triplicate $(n=3)$, except for mass flow rate, which was measured in duplicate $(n=2)$. The results were analyzed via Statistical Analysis System (SAS) software, which determined the means and standard deviations for each treatment, as well as the main effects, interactions, and treatment combination effects, using an $\alpha=0.05$. 
Table 1. Experimental design*

\begin{tabular}{cccc}
\hline Treatment & Screw Speed $(\mathrm{rpm})$ & Moisture Content $(\% \mathrm{db})$ & Temperature $\left({ }^{\circ} \mathrm{C}\right)$ \\
\hline 1 & 50 & 15 & 100 \\
2 & 50 & 15 & 150 \\
3 & 50 & 35 & 100 \\
4 & 50 & 35 & 150 \\
5 & 100 & 15 & 100 \\
6 & 100 & 15 & 150 \\
7 & 100 & 35 & 100 \\
8 & 100 & 35 & 150 \\
\hline 9 (Center Point) & 75 & 25 & 125 \\
\hline
\end{tabular}

*The run value describes the order each sample was extruded. Experimental design was a $2 \times 2 \times 2+1$ center point $=9$ treatment conditions. The Center Point was defined as Treatment 9.

\section{Results and Discussion}

With the extrusion conditions used in the study, neither the corn nor the DDGS extrudates were of exceptionally high quality, unfortunately. As shown in Figure 1, the DDGS extrudates did not exhibit extensive particle binding during processing, which led to pellets which were not highly cohesive. (Images of corn extrudates are not provided, but exhibited similar behavior.) It is likely that increasing the moisture of the raw blends could have improved particle binding, and thus pellet quality. It should be noted that in subsequent tables that some of the extrusion conditions did not produce results - this occurred due to starch gelatinization within the extruder barrel, which caused the screw to foul, and thus extrusion had to be discontinued.
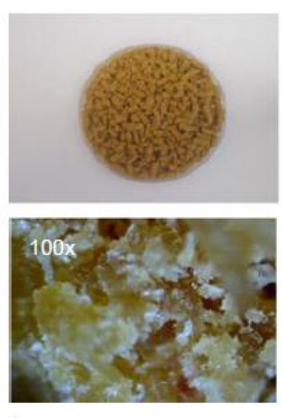

1
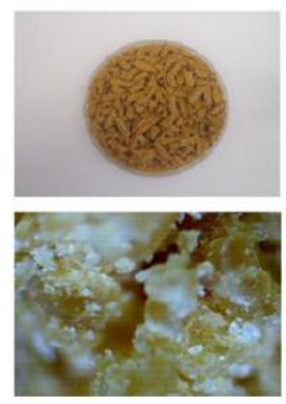
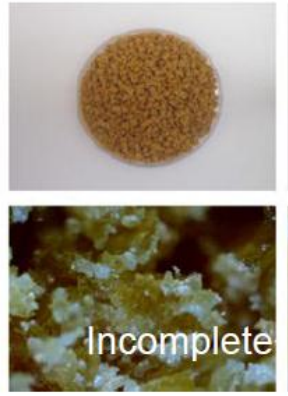

2
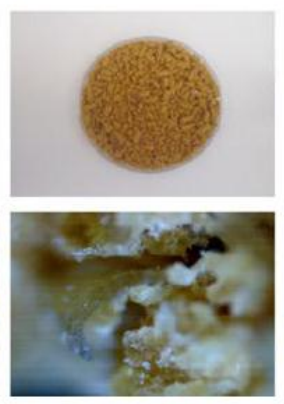

7
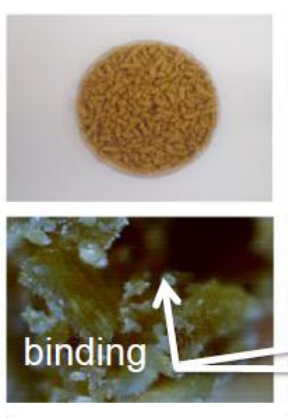

3
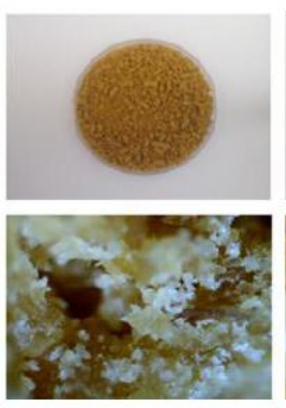
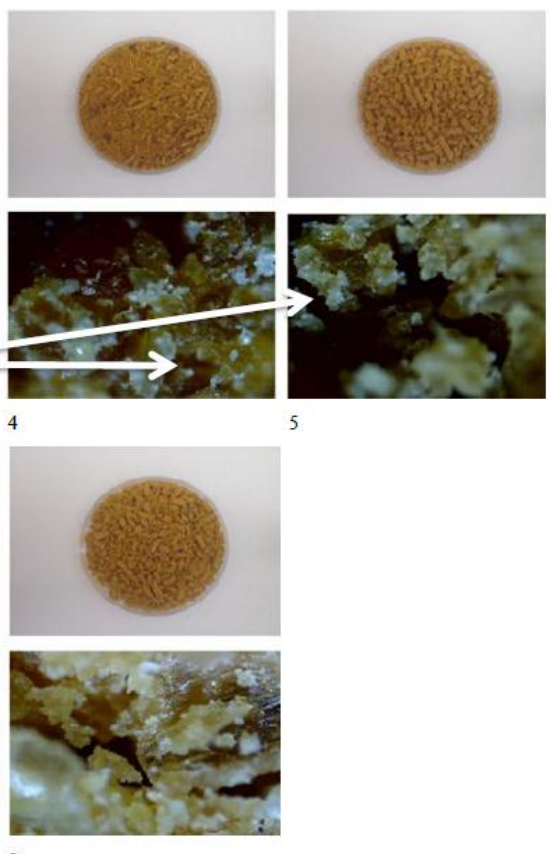

Figure 1. Bulk and cross-sectional (100x) images of the DDGS extrudates for each treatment (1-9). Image analysis indicates that the quality of the extrudates was low due to incomplete particle binding which occurred during all extrusion conditions used in this study.

\subsection{Raw Ingredient Properties}

Table 2 and Table 3 provide the results of the effects of moisture content on the water activity and color ( $\mathrm{L}^{*}$, $\left.a^{*}, b^{*}\right)$ of the DDGS and corn, respectively. 
Table 2. Raw DDGS properties*

\begin{tabular}{cccccc}
\hline \multirow{2}{*}{ Treatment } & $\begin{array}{c}\text { Moisture Content } \\
(\mathrm{db}, \%)\end{array}$ & $\begin{array}{c}\text { Water Activity } \\
\left(\mathrm{a}_{\mathrm{w}}\right)\end{array}$ & $\begin{array}{c}\text { Color } \\
\mathrm{L}^{*}\end{array}$ & $\begin{array}{c}\mathrm{a}^{*} \\
\mathrm{~b}^{*}\end{array}$ \\
\hline \multirow{2}{*}{1} & 15.20 & 0.61 & 58.92 & 14.82 & 48.48 \\
& $(1.11)$ & $(0.00)$ & $(0.95)$ & $(0.28)$ & $(0.08)$ \\
2 & 15.20 & 0.60 & 58.92 & 14.82 & 48.48 \\
& $(1.11)$ & $(0.00)$ & $(0.95)$ & $(0.28)$ & $(0.08)$ \\
3 & 33.78 & 0.83 & 58.92 & 14.82 & 48.48 \\
& $(0.71)$ & $(0.00)$ & $(0.95)$ & $(0.28)$ & $(0.08)$ \\
4 & 33.78 & 0.83 & 58.92 & 14.82 & 48.48 \\
& $(0.71)$ & $(0.00)$ & $(0.95)$ & $(0.28)$ & $(0.08)$ \\
5 & 15.20 & 0.60 & 58.92 & 14.82 & 48.48 \\
& $(1.11)$ & $(0.00)$ & $(0.95)$ & $(0.28)$ & $(0.08)$ \\
& 15.20 & 0.60 & 58.92 & 14.82 & 48.48 \\
6 & $(1.11)$ & $(0.00)$ & $(0.95)$ & $(0.28)$ & $(0.08)$ \\
& 33.78 & 0.83 & 58.92 & 14.82 & 48.48 \\
7 & $(0.71)$ & $(0.00)$ & $(0.95)$ & $(0.28)$ & $(0.08)$ \\
& 33.78 & 0.83 & 58.92 & 14.82 & 48.48 \\
8 & $(0.71)$ & $(0.00)$ & $(0.95)$ & $(0.28)$ & $(0.08)$ \\
& 23.95 & 0.75 & 58.92 & 14.82 & 48.48 \\
9 & $(0.61)$ & $(0.00)$ & $(0.95)$ & $(0.28)$ & $(0.08)$ \\
\hline \multirow{2}{*}{5} & & & & & \\
& & &
\end{tabular}

* Averages and standard deviations (shown in parentheses) for various physical properties of raw DDGS samples.

Table 3. Raw corn properties *

\begin{tabular}{cccccc}
\hline Treatment & $\begin{array}{c}\text { Moisture Content } \\
(\mathrm{db}, \%)\end{array}$ & $\begin{array}{c}\text { Water Activity } \\
\left(\mathrm{a}_{\mathrm{w}}\right)\end{array}$ & $\begin{array}{c}\text { Color } \\
\mathrm{L}^{*}\end{array}$ & $\mathrm{a}^{*}$ & $\mathrm{~b}^{*}$ \\
\hline \multirow{2}{*}{1} & 16.16 & - & 80.66 & 5.20 & 32.41 \\
& $(0.77)$ & - & $(1.18)$ & $(0.20)$ & $(1.49)$ \\
2 & 16.16 & - & 80.66 & 5.20 & 32.41 \\
& $(0.77)$ & - & $(1.18)$ & $(0.20)$ & $(1.49)$ \\
3 & 34.26 & 0.97 & 80.66 & 5.20 & 32.41 \\
& $(0.66)$ & $(0.00)$ & $(1.18)$ & $(0.20)$ & $(1.49)$ \\
4 & 34.26 & - & 80.66 & 5.20 & 32.41 \\
& $(0.66)$ & - & $(1.18)$ & $(0.20)$ & $(1.49)$ \\
5 & 16.16 & 0.75 & 80.66 & 5.20 & 32.41 \\
& $(0.77)$ & $(0.00)$ & $(1.18)$ & $(0.20)$ & $(1.49)$ \\
& 16.16 & 0.76 & 80.66 & 5.20 & 32.41 \\
6 & $(0.77)$ & $(0.00)$ & $(1.18)$ & $(0.20)$ & $(1.49)$ \\
& 34.26 & 0.98 & 80.66 & 5.20 & 32.41 \\
7 & $(0.66)$ & $(0.00)$ & $(1.18)$ & $(0.20)$ & $(1.49)$ \\
& 34.26 & 0.98 & 80.66 & 5.20 & 32.41 \\
8 & $(0.66)$ & $(0.00)$ & $(1.18)$ & $(0.20)$ & $(1.49)$ \\
& 23.68 & - & 80.66 & 5.20 & 32.41 \\
9 & $(0.47)$ & - & $(1.18)$ & $(0.20)$ & $(1.49)$ \\
\hline
\end{tabular}

*Averages and standard deviations (shown in parentheses) for various physical properties of raw corn samples.

\subsubsection{Moisture Content}

According to Table 2, the exact moisture contents were relatively close to the desired moisture content of the raw DDGS. The exact moisture content values were off by $1.34,4.40$, and $3.62 \%$ for the targets of 15,25 , and $35 \%$, $\mathrm{db}$, respectively. Because of the low variance, the extrusion process was performed without further adjustment.

\subsubsection{Water Activity}

The water activity level of the raw ingredients is important because the greater the value, the greater the chance for microbial metabolism and growth. The greater the microbial growth rate, the more likely the product is to spoil. According to Table 2, as the moisture content increased, the water activity level increased in the raw DDGS blends. Similar results are seen in Table 3 for the raw corn blends.

\subsection{Extrudate Properties}

Table 4 and Table 5 provide the results for the treatment effects of moisture content, screw speed, and temperature on the moisture content, water activity, color $\left(L^{*}, a^{*}, b^{*}\right)$, unit density, pellet durability, water stability, floatability, and bulk density of the DDGS and corn extrudates, respectively. The tables display the 
averages for each property, as well as the standard deviations, which are shown in parentheses. Table 6 displays the means and standard deviations for the main effects on the dependent variables of the DDGS extrudates. Table 7 displays the interaction effects.

Table 4. Treatment effects on properties of DDGS extrudates*

\begin{tabular}{|c|c|c|c|c|c|c|c|c|c|c|}
\hline Treatment & $\begin{array}{c}\text { Moisture Content } \\
(\mathrm{db} \%)\end{array}$ & $\begin{array}{c}\text { Water Activity } \\
\left(\mathrm{a}_{\mathrm{w}}\right)\end{array}$ & $\mathrm{L}^{*}$ & $\begin{array}{c}\text { Color } \\
a^{*}\end{array}$ & $b^{*}$ & Unit Density & $\begin{array}{c}\text { Pellet Durability } \\
\text { Index }(\%)\end{array}$ & $\begin{array}{c}\text { Water } \\
\text { Stability (s) }\end{array}$ & Floatability & Bulk Density \\
\hline 1 & $\begin{array}{c}15.52 \mathrm{~cd} \\
(0.29)\end{array}$ & $\begin{array}{l}0.63 \mathrm{c} \\
(0.01)\end{array}$ & $\begin{array}{c}52.56 \mathrm{abc} \\
(1.72)\end{array}$ & $\begin{array}{l}12.57 \mathrm{~b} \\
(0.18)\end{array}$ & $\begin{array}{l}41.10 \mathrm{bc} \\
(0.67)\end{array}$ & $\begin{array}{l}0.72 \mathrm{ab} \\
(0.05)\end{array}$ & $\begin{array}{l}38.83 \mathrm{bc} \\
(2.27)\end{array}$ & $\begin{array}{c}4.00 \mathrm{de} \\
(0.0)\end{array}$ & $\begin{array}{c}2.67 \mathrm{abc} \\
(0.58)\end{array}$ & $378.80 \mathrm{bc}$ \\
\hline 2 & $\begin{array}{c}15.32 \mathrm{~cd} \\
(0.81)\end{array}$ & $\begin{array}{l}0.63 \mathrm{c} \\
(0.0)\end{array}$ & $\begin{array}{l}50.45 \mathrm{c} \\
(1.36)\end{array}$ & $\begin{array}{l}14.02 \mathrm{a} \\
(0.33)\end{array}$ & $\begin{array}{l}41.13 \mathrm{bc} \\
(0.13)\end{array}$ & $\begin{array}{r}0.75 \mathrm{ab} \\
(0.11)\end{array}$ & $\begin{array}{c}34.87 \mathrm{~cd} \\
(1.60)\end{array}$ & $\begin{array}{l}8.00 \mathrm{~b} \\
(1.00)\end{array}$ & $\begin{array}{l}3.33 \mathrm{a} \\
(0.58)\end{array}$ & $\begin{array}{c}372.00 \mathrm{c} \\
(4.93)\end{array}$ \\
\hline 3 & $\begin{array}{c}18.80 \mathrm{~b} \\
(0.37)\end{array}$ & $\begin{array}{c}0.70 \mathrm{a} \\
(0.0)\end{array}$ & $\begin{array}{l}52.34 \mathrm{bc} \\
(0.93)\end{array}$ & $\begin{array}{l}12.70 \mathrm{~b} \\
(0.50)\end{array}$ & $\begin{array}{c}40.03 \mathrm{c} \\
(0.95)\end{array}$ & $\begin{array}{r}0.78 \mathrm{ab} \\
(0.08)\end{array}$ & $\begin{array}{l}40.60 \mathrm{bc} \\
(7.59)\end{array}$ & $\begin{array}{l}6.00 \mathrm{c} \\
(1.00)\end{array}$ & $\begin{array}{l}2.00 \mathrm{c} \\
(0.0)\end{array}$ & $\begin{array}{l}399.00 \mathrm{a} \\
(2.16)\end{array}$ \\
\hline 4 & $\begin{array}{l}16.03 \mathrm{c} \\
(0.33)\end{array}$ & $\begin{array}{l}0.62 \mathrm{c} \\
(0.01)\end{array}$ & $\begin{array}{l}53.49 \mathrm{ab} \\
(0.94)\end{array}$ & $\begin{array}{l}14.23 \mathrm{a} \\
(0.39)\end{array}$ & $\begin{array}{r}44.45 \mathrm{a} \\
(1.24)\end{array}$ & $\begin{array}{l}0.85 \mathrm{a} \\
(0.04)\end{array}$ & $\begin{array}{l}- \\
-\end{array}$ & $\begin{array}{l}1.67 \mathrm{fg} \\
(0.58)\end{array}$ & $\begin{array}{l}3.33 \mathrm{a} \\
(0.58)\end{array}$ & $\begin{array}{l}- \\
-\end{array}$ \\
\hline 5 & $\begin{array}{c}15.28 \mathrm{~cd} \\
(0.29)\end{array}$ & $\begin{array}{l}0.65 \mathrm{~b} \\
(0.02)\end{array}$ & $\begin{array}{l}52.72 \mathrm{abc} \\
(1.21)\end{array}$ & $\begin{array}{l}12.92 \mathrm{~b} \\
(0.16)\end{array}$ & $\begin{array}{c}41.96 b \\
(0.52)\end{array}$ & $\begin{array}{l}0.62 \mathrm{~b} \\
(0.03)\end{array}$ & $\begin{array}{c}36.53 \mathrm{~cd} \\
(1.12)\end{array}$ & $\begin{array}{r}3.67 \mathrm{de} \\
(0.58)\end{array}$ & $\begin{array}{r}2.33 \mathrm{bc} \\
(0.58)\end{array}$ & $\begin{array}{l}355.00 \mathrm{~d} \\
(7.34)\end{array}$ \\
\hline 6 & $\begin{array}{l}14.58 \mathrm{~d} \\
(0.32)\end{array}$ & $\begin{array}{l}0.63 \mathrm{c} \\
(0.01)\end{array}$ & $\begin{array}{l}52.20 \mathrm{bc} \\
(2.59)\end{array}$ & $\begin{array}{l}12.77 \mathrm{~b} \\
(0.16)\end{array}$ & $\begin{array}{c}40.25 \mathrm{c} \\
(0.20)\end{array}$ & $\begin{array}{l}0.86 \mathrm{a} \\
(0.31)\end{array}$ & $\begin{array}{l}47.90 \mathrm{a} \\
(1.71)\end{array}$ & $\begin{array}{c}5.00 \mathrm{~cd} \\
(1.00)\end{array}$ & $\begin{array}{c}3.00 \mathrm{ab} \\
(0.0)\end{array}$ & $\begin{array}{c}324.73 \mathrm{e} \\
(6.82)\end{array}$ \\
\hline 7 & $\begin{array}{c}20.53 \mathrm{a} \\
(2.15)\end{array}$ & $\begin{array}{l}0.68 \mathrm{a} \\
(0.03)\end{array}$ & $\begin{array}{c}55.35 \mathrm{a} \\
(2.11)\end{array}$ & $\begin{array}{l}13.82 \mathrm{a} \\
(0.29)\end{array}$ & $\begin{array}{c}43.81 \mathrm{a} \\
(0.94)\end{array}$ & $\begin{array}{r}0.69 \mathrm{ab} \\
(0.09)\end{array}$ & $\begin{array}{r}30.63 d \\
(4.68)\end{array}$ & $\begin{array}{l}3.00 \mathrm{ef} \\
(1.00)\end{array}$ & $\begin{array}{c}2.67 \mathrm{abc} \\
(0.58)\end{array}$ & $\begin{array}{c}381.80 \mathrm{~b} \\
(0.20)\end{array}$ \\
\hline 8 & $\begin{array}{c}18.82 \mathrm{~b} \\
(0.28)\end{array}$ & $\begin{array}{l}0.69 \mathrm{a} \\
(0.01)\end{array}$ & $\begin{array}{c}55.06 \mathrm{ab} \\
(1.96)\end{array}$ & $\begin{array}{l}13.89 \mathrm{a} \\
(0.15)\end{array}$ & $\begin{array}{c}44.32 \mathrm{a} \\
(1.48)\end{array}$ & $\begin{array}{r}0.84 \mathrm{ab} \\
(0.13)\end{array}$ & $\begin{array}{l}17.33 \mathrm{e} \\
(6.05)\end{array}$ & $\begin{array}{r}0.40 \mathrm{~g} \\
(0.0)\end{array}$ & $\begin{array}{c}2.67 \mathrm{abc} \\
(0.58)\end{array}$ & $\begin{array}{c}385.53 \mathrm{~b} \\
(1.29)\end{array}$ \\
\hline
\end{tabular}

Averages and standard deviations (shown in parentheses) for various physical properties of DDGS extrudates. The treatment effect relationships are shown below. Differing letters indicate significant differences among treatments $(\alpha=0.05)$ for a given dependent variable.

Table 5. Treatment effects on properties of corn extrudates*

\begin{tabular}{|c|c|c|c|c|c|c|c|c|c|c|}
\hline Treatment & $\begin{array}{c}\text { Moisture Content } \\
(\mathrm{db}, \%)\end{array}$ & $\begin{array}{c}\text { Water Activity } \\
\left(\mathrm{a}_{\mathrm{w}}\right)\end{array}$ & $\mathrm{L}^{*}$ & $\begin{array}{c}\text { Color } \\
a^{*}\end{array}$ & $b^{*}$ & $\begin{array}{c}\text { Unit Density } \\
\left(\mathrm{g} / \mathrm{cm}^{3}\right)\end{array}$ & $\begin{array}{l}\text { Pellet Durability } \\
\text { Index }(\%)\end{array}$ & $\begin{array}{c}\text { Water } \\
\text { Stability (s) }\end{array}$ & $\begin{array}{c}\text { Floatability } \\
\text { (s) }\end{array}$ & $\begin{array}{c}\text { Bulk Density } \\
\left(\mathrm{kg} / \mathrm{m}^{3}\right)\end{array}$ \\
\hline \multirow{2}{*}{1} & - & - & - & - & - & - & - & - & - & - \\
\hline & - & - & - & - & - & - & - & - & - & - \\
\hline \multirow{2}{*}{2} & - & - & - & - & - & - & - & - & - & - \\
\hline & - & - & - & - & - & - & - & - & - & - \\
\hline \multirow{2}{*}{3} & 11.39 & 0.65 & 46.66 & 10.81 & 40.93 & 1.32 & 99.80 & 1800.00 & $<1$ & 620.33 \\
\hline & (0.96) & $(0.01)$ & (1.96) & (0.89) & (1.13) & $(0.03)$ & (0.10) & $(0.0)$ & $(0.0)$ & (1.70) \\
\hline \multirow{2}{*}{4} & - & - & - & - & - & - & - & - & - & - \\
\hline & - & - & - & - & - & - & - & - & - & - \\
\hline \multirow{2}{*}{5} & 12.69 & 0.65 & 62.54 & 4.90 & 35.06 & 1.14 & 97.73 & 1729.00 & $<1$ & 490.07 \\
\hline & (0.13) & $(0.0)$ & (2.31) & $(0.41)$ & $(1.28)$ & $(0.08)$ & (0.15) & (67.77) & $(0.0)$ & (5.90) \\
\hline \multirow{2}{*}{6} & 12.89 & 0.63 & 66.39 & 3.57 & 31.37 & 0.78 & - & 468.00 & 902.67 & - \\
\hline & $(0.05)$ & (0.0) & (1.81) & $(0.32)$ & $(0.95)$ & $(0.05)$ & - & (44.00) & (85.80) & - \\
\hline \multirow{2}{*}{7} & 15.42 & 0.72 & 52.47 & 8.99 & 40.78 & 1.31 & 99.40 & 1800.00 & $<1$ & 599.20 \\
\hline & (5.35) & $(0.04)$ & (0.90) & $(0.33)$ & $(1.22)$ & (0.09) & $(0.70)$ & $(0.0)$ & $(0.0)$ & (3.14) \\
\hline \multirow{2}{*}{8} & 14.41 & 0.65 & 57.99 & 6.16 & 31.40 & 1.12 & 98.90 & 1800.00 & 0.33 & 446.87 \\
\hline & $(0.24)$ & $(0.01)$ & (2.04) & $(0.88)$ & $(1.62)$ & $(0.08)$ & $(0.10)$ & $(0.0)$ & $(0.0)$ & (1.68) \\
\hline \multirow{2}{*}{9} & - & - & - & - & - & - & - & - & - & - \\
\hline & - & - & - & - & - & - & - & - & - & - \\
\hline
\end{tabular}

* Averages and standard deviations (shown in parentheses) for various physical properties of corn extrudates.

Table 6. Main effects on properties of DDGS extrudates* 


\begin{tabular}{|c|c|c|c|c|c|c|c|c|c|c|c|}
\hline Parameter & Levels & $\begin{array}{c}\text { Moisture Content } \\
(\% \mathrm{db})\end{array}$ & $\begin{array}{c}\text { Water Activity } \\
\left(a_{w}\right)\end{array}$ & $\mathrm{L}^{*}$ & $\begin{array}{c}\text { Color } \\
\mathrm{a}^{*} \\
\end{array}$ & $b^{*}$ & $\begin{array}{l}\text { Unit Density } \\
\left(\mathrm{g} / \mathrm{cm}^{3}\right)\end{array}$ & $\begin{array}{c}\text { Pellet Durability } \\
\text { Index }(\%)\end{array}$ & $\begin{array}{c}\text { Water Stability } \\
(\mathrm{s})\end{array}$ & $\begin{array}{c}\text { Floatability } \\
(\mathrm{s})\end{array}$ & $\begin{array}{c}\text { Bulk Density } \\
\left(\mathrm{kg} / \mathrm{m}^{3}\right)\end{array}$ \\
\hline \multirow{6}{*}{$\begin{array}{l}\text { Moisture } \\
\text { Content }(\% \\
\text { db) }\end{array}$} & \multirow{2}{*}{15} & $15.18 \mathrm{a}$ & $0.64 \mathrm{a}$ & $51.99 \mathrm{a}$ & $13.07 \mathrm{a}$ & $41.11 \mathrm{a}$ & $0.74 \mathrm{a}$ & $39.53 \mathrm{a}$ & $5.17 \mathrm{a}$ & $2.83 a$ & $357.63 a$ \\
\hline & & $(0.55)$ & $(0.01)$ & $(1.80)$ & $(0.62)$ & $(0.74)$ & $(0.17)$ & (5.46) & (1.90) & (0.58) & $(22.32)$ \\
\hline & \multirow{2}{*}{25} & $16.28 \mathrm{~b}$ & $0.64 a$ & $52.43 \mathrm{ab}$ & $12.64 \mathrm{~b}$ & $40.77 \mathrm{a}$ & $0.75 a$ & $43.83 a$ & $9.67 \mathrm{~b}$ & $2.00 \mathrm{~b}$ & $359.33 \mathrm{a}$ \\
\hline & & (0.17) & $(0.01)$ & (1.65) & $(0.28)$ & $(0.18)$ & $(0.11)$ & (2.68) & (1.15) & $(0.0)$ & (0.99) \\
\hline & \multirow{2}{*}{35} & $18.55 \mathrm{c}$ & $0.68 \mathrm{~b}$ & $54.06 \mathrm{~b}$ & $13.66 \mathrm{c}$ & $43.15 b$ & $0.79 \mathrm{a}$ & $29.52 b$ & $2.77 \mathrm{c}$ & $2.67 \mathrm{a}$ & $388.78 \mathrm{~b}$ \\
\hline & & (1.94) & $(0.03)$ & $(1.86)$ & $(0.67)$ & (2.15) & (0.10) & (11.45) & $(2.28)$ & $(0.65)$ & (7.94) \\
\hline \multirow{6}{*}{$\begin{array}{c}\text { Temperature } \\
\left({ }^{\circ} \mathrm{C}\right)\end{array}$} & \multirow[b]{2}{*}{100} & $17.54 a$ & $0.67 \mathrm{a}$ & $53.24 \mathrm{a}$ & $13.00 \mathrm{a}$ & $41.72 \mathrm{a}$ & $0.70 \mathrm{a}$ & $36.65 \mathrm{a}$ & $4.17 \mathrm{a}$ & $2.42 \mathrm{a}$ & $378.65 \mathrm{a}$ \\
\hline & & $(2.50)$ & $(0.03)$ & $(1.85)$ & $(0.57)$ & $(1.60)$ & $(0.08)$ & (5.57) & (1.34) & $(0.51)$ & (16.70) \\
\hline & \multirow{2}{*}{125} & $16.27 \mathrm{~b}$ & $0.64 \mathrm{~b}$ & $52.43 \mathrm{a}$ & $12.64 \mathrm{a}$ & $40.77 \mathrm{a}$ & $0.75 a b$ & $43.83 \mathrm{~b}$ & $9.67 \mathrm{~b}$ & $2.00 \mathrm{a}$ & $359.33 \mathrm{~b}$ \\
\hline & & $(0.17)$ & $(0.01)$ & $(1.65)$ & $(0.28)$ & $(0.18)$ & $(0.11)$ & (2.68) & (1.15) & $(0.0)$ & $(0.99)$ \\
\hline & \multirow{2}{*}{150} & $16.19 \mathrm{~b}$ & $0.64 \mathrm{~b}$ & $52.80 \mathrm{a}$ & $13.73 \mathrm{~b}$ & $42.54 \mathrm{~b}$ & $0.82 \mathrm{~b}$ & $33.37 \mathrm{a}$ & $3.77 \mathrm{a}$ & $3.08 \mathrm{~b}$ & $360.76 \mathrm{~b}$ \\
\hline & & (1.73) & $(0.03)$ & $(2.35)$ & $(0.64)$ & (2.13) & $(0.16)$ & (13.67) & (3.17) & $(0.51)$ & (27.97) \\
\hline \multirow{6}{*}{$\begin{array}{l}\text { Screw Speed } \\
\text { (rpm) }\end{array}$} & \multirow[b]{2}{*}{50} & $16.42 \mathrm{a}$ & $0.65 \mathrm{a}$ & $52.21 \mathrm{a}$ & $13.38 \mathrm{a}$ & $41.68 \mathrm{a}$ & $0.77 \mathrm{a}$ & $38.10 \mathrm{a}$ & $4.92 \mathrm{a}$ & $2.83 a$ & $383.27 \mathrm{a}$ \\
\hline & & (1.52) & $(0.03)$ & $(1.59)$ & $(0.84)$ & (1.88) & $(0.08)$ & (4.78) & (2.54) & $(0.72)$ & (12.46) \\
\hline & \multirow{2}{*}{75} & $16.28 \mathrm{ab}$ & $0.64 \mathrm{a}$ & $52.43 \mathrm{ab}$ & $12.64 \mathrm{~b}$ & $40.77 \mathrm{a}$ & $0.75 \mathrm{a}$ & $43.83 \mathrm{a}$ & $9.67 \mathrm{~b}$ & $2.00 \mathrm{~b}$ & $359.33 \mathrm{~b}$ \\
\hline & & $(0.17)$ & $(0.01)$ & $(1.65)$ & $(0.28)$ & $(0.18)$ & (0.11) & (2.68) & (1.15) & $(0.0)$ & $(0.99)$ \\
\hline & \multirow{2}{*}{100} & $17.31 \mathrm{~b}$ & $0.67 \mathrm{~b}$ & $53.84 \mathrm{~b}$ & $13.35 \mathrm{a}$ & $42.58 \mathrm{~b}$ & $0.75 a$ & $33.10 \mathrm{~b}$ & $3.02 \mathrm{c}$ & $2.67 \mathrm{a}$ & $361.77 \mathrm{~b}$ \\
\hline & & (2.74) & $(0.03)$ & $(2.26)$ & $(0.56)$ & $(1.85)$ & $(0.18)$ & (11.99) & $(1.88)$ & $(0.49)$ & $(25.86)$ \\
\hline
\end{tabular}

*Averages and standard deviations (shown in parentheses) of the main effects of the DDGS extrudates. The relationships amongst the main effects are shown below. Differing letters indicate significant differences among levels of a specific independent variable $(\alpha=0.05)$ for a given dependent variable.

Table 7. Interaction effects for properties of DDGS extrudates*

\begin{tabular}{|c|c|c|c|c|c|}
\hline Parameter & $\begin{array}{c}\text { Moisture Content } \\
(\% \mathrm{db})\end{array}$ & $\begin{array}{c}\text { Water Activity } \\
(\mathrm{aw})\end{array}$ & $\mathrm{L}^{*}$ & $\begin{array}{c}\text { Color } \\
a^{*}\end{array}$ & $\mathrm{~b}^{*}$ \\
\hline Moisture Content & $<.0001$ & $<.0001$ & 0.0076 & 0.0001 & $<.0001$ \\
\hline Temperature & 0.0007 & 0.0001 & 0.5319 & $<.0001$ & 0.0285 \\
\hline Screw Speed & 0.0152 & 0.0017 & 0.0301 & 0.7940 & 0.0163 \\
\hline Moisture Content*Temperature & 0.0145 & 0.0173 & 0.2219 & 0.5380 & 0.0001 \\
\hline Moisture Content* Screw Speed & 0.0006 & 0.2090 & 0.3458 & 0.0024 & 0.0154 \\
\hline Temperature* Screw Speed & 0.6696 & 0.0043 & 0.9582 & $<.0001$ & 0.0006 \\
\hline Moisture Content*Temperature* Screw Speed & 0.2523 & $<.0001$ & 0.2874 & 0.7624 & 0.1321 \\
\hline Parameter & Unit Density $\left(\mathrm{g} / \mathrm{cm}^{3}\right)$ & $\begin{array}{l}\text { Pellet Durability } \\
\text { Index }(\%)\end{array}$ & $\begin{array}{c}\text { Water } \\
\text { Stability (s) }\end{array}$ & $\begin{array}{c}\text { Floatability } \\
\text { (s) }\end{array}$ & $\begin{array}{c}\text { Bulk Density } \\
\left(\mathrm{kg} / \mathrm{m}^{3}\right)\end{array}$ \\
\hline Moisture Content & 0.3165 & $<.0001$ & $<.0001$ & 0.3979 & $<.0001$ \\
\hline Temperature & 0.0382 & 0.0352 & 0.2557 & 0.0028 & 0.0022 \\
\hline Screw Speed & 0.6934 & 0.7084 & $<.0001$ & 0.3979 & $<.0001$ \\
\hline Moisture Content* Temperature & 0.8308 & $<.0001$ & $<.0001$ & 1.0000 & $<.0001$ \\
\hline Moisture Content*Screw Speed & 0.6489 & 0.1258 & 0.5022 & 0.3979 & 0.1793 \\
\hline Temperature* Screw Speed & 0.1904 & 0.0052 & 0.5022 & 0.1004 & 0.0001 \\
\hline Moisture Content*Temperature* Screw Speed & 0.5479 & - & 0.0047 & 0.1004 & - \\
\hline
\end{tabular}

* Relationships between the treatment interactions of the DDGS extrudates (p-values). Interactions were deemed significant if $\mathrm{p}<0.05$.

\subsubsection{Moisture Content}

The moisture content of the extrudates is important to consider because it determines the freshness of the pellets. Moisture also impacts the rate of spoilage of the extrudates. When developing a pellet for aquaculture feed, it is important to consider the amount of time the pellets will be stored, so the optimum amount of moisture present in the extrudates can be determined. According to Table 4, treatment 7, which had a screw speed of $100 \mathrm{rpm}$, raw moisture content of $35 \% \mathrm{db}$, and die temperature of $100^{\circ} \mathrm{C}$, had the greatest extrudate moisture content and was significantly different from the other eight treatments. Treatments 3 and 8 had similar moisture contents of $18.80 \%$ $\mathrm{db}$ and $18.82 \% \mathrm{db}$, respectively, and treatments 4 and 9 were similar as well, with moisture contents of $16.03 \%$ $\mathrm{db}$ and $16.28 \% \mathrm{db}$, respectively. Treatment 6 , which had a screw speed of $100 \mathrm{rpm}$, raw moisture content of $15 \%$ $\mathrm{db}$, and a die temperature of $150^{\circ} \mathrm{C}$, had the lowest extrudate moisture content of $14.58 \% \mathrm{db}$. Treatments 1,2 , and 5 had similar results to both treatments 4 and 9 and treatment 6 , with extrudate moisture contents of $15.52 \%$ $\mathrm{db}, 15.32 \% \mathrm{db}$, and $15.28 \% \mathrm{db}$, respectively. According to Table 3, the greater the initial moisture content of the raw DDGS blends, the greater the moisture content in the extrudates. A similar relationship between the corn extrudates was determined as well. According to Table 6, as the temperature of the die increased, the moisture content of the DDGS extrudates decreased, due to increased evaporation. According to Table 6, as the screw 
speed of the extruder increased from 50 to $100 \mathrm{rpm}$, the moisture content of the DDGS extrudates increased. According to Table 5, the amount of moisture present in the corn extrudates increased as the screw speed of the extruder increased; this was similar behavior as the DDGS.

\subsubsection{Water Activity}

Water activity determines the amount of water available for microbial metabolism and growth and is important in the extrudates because it determines the rate of spoilage. When comparing moisture content to water activity, the water activity is more important in the biological sense of spoilage because it is the amount of water available for microbial growth. According to Table 4, treatments 3, 7, and 8 have the greatest water activity levels, with values of $0.70,0.68$, and 0.69 , respectively. With a value of 0.65 , treatment 5 has a slightly smaller water activity value and is significantly different than treatments 3,7 , and 8 . Treatments $1,2,4$, and 6 have the smallest values for water activity, and treatment 9 has a value of 0.64 , which is not significantly different from treatments $1,2,4,5$, and 6. According to Table 6, as the moisture content of the raw DDGS ingredients increased, the water activity of the extrudates increased. A similar relationship as seen in the corn extrudates and is shown in Table 5. As the temperature of the die of the extruder increased, the water activity in the DDGS extrudates decreased and is displayed in Table 6. Similar results are shown in Table 5 for the corn extrudates. As the screw speed of the extruder increased, there was an increase in water activity levels from the 50 and $75 \mathrm{rpm}$ to the $100 \mathrm{rpm}$ treatments. According to Table 5, as the screw speed of the extruder increased, the water activity level of the corn extrudates increased.

\subsubsection{Color}

In order to have consumer acceptance, the appearance of extrudates is important. Color is one of the most important appearance attributes because consumers buy products based on expected color from previous experience. The color of the extrudates is measured based on three parameters: $\mathrm{L}^{*}, \mathrm{a}^{*}$, and $\mathrm{b}^{*}$. According to Table 4, the $\mathrm{L}^{*}$ value for the DDGS was greatest in treatment 7 , with a value of 55.35 , while treatment 2 had the smallest $\mathrm{L}^{*}$ value at 50.45 . Treatments $2,4,7$, and 8 have higher $\mathrm{a}^{*}$ values that are significantly different from treatments $1,3,5,6$, and 9 (Table 4). The $\mathrm{b}^{*}$ values for treatments 4,7 , and 8 were the greatest and were significantly different from the other treatments. Treatments 3 and 6 have the lowest $b^{*}$ values of 40.03 and 40.25 , respectively. As the moisture content of the DDGS extrudates increased, the $\mathrm{L}^{*}$ and $\mathrm{b}^{*}$ values increased, although there was no relationship with $a^{*}$ values, according to Table 6 . A similar relationship was true for the corn extrudates for the $b^{*}$ values (Table 5). As the temperature of the die increased, the $a^{*}$ and $b^{*}$ values of the DDGS extrudates increased, but there was no relationship with the $\mathrm{L}^{*}$ values. As the screw speed of the extruder increased, the $\mathrm{L}^{*}$ and $\mathrm{b}^{*}$ values of the DDGS extrudates increased, but there was not a significant relationship with the $\mathrm{a}^{*}$ values.

\subsubsection{Unit Density}

Unit density is important in the extrudates because the amount of mass per unit volume should contain the proper nutrients, in the proper quantities but be able to float in water (i.e., if UD $<1.0 \mathrm{~g} / \mathrm{cm}^{3}$ ). According to Table 4 , treatment 4 and 6 contained the greatest mass per unit volume, with values of 0.85 and $0.86 \mathrm{~g} / \mathrm{cm}^{3}$, respectively, while treatment 5 produced the smallest unit density with a value of $0.62 \mathrm{~g} / \mathrm{cm}^{3}$. Treatments $1,2,3,7,8$, and 9 were similar to treatments 4,5 , and 6 . According to Table 6 , there was no significant relationship between the unit density of the DDGS extrudates and the change in moisture content or screw speed. However, as the temperature of the die increased, the unit density of the DDGS extrudates increased. Because all treatments had UD values less than $1.0 \mathrm{~g} / \mathrm{cm}^{3}$, they should have been able to float in water (if they could exhibit water stability, that is).

\subsubsection{Pellet Durability Index}

Pellet durability index is the amount of breakage the extrudate is able to withstand. It is important in aquaculture feeds because of storage and delivery processes. According to Table 4, the greatest value for the pellet durability index was treatment 6 at $47.90 \%$. Treatments 1 and 3 were relatively similar to both treatment 9 , as well as treatments 2 and 5. Treatment 7 was similar to treatments 2 and 5 as well. The lowest pellet durability index was treatment 8 , at $17.33 \%$. The pellet durability index for treatment 4 was unable to be determined due to the lack of cohesive of extrudates for this treatment, thus PDI could not be reported. According to Table 6, as the moisture content of the DDGS extrudates increased, the pellet durability index decreased. There was no significant relationship between the temperature of the DDGS extrudates and the pellet durability index values. As the screw speed of the extruder increased, the pellet durability index decreased in the DDGS extrudates.

\subsubsection{Water Stability}


Water stability is the amount of time the pellet holds together in water before it dissolves. It is important for aquaculture feeds because the fish need pellets to last approximately 30 min during feeding time. According to Table 4 , the greatest value for water stability was treatment 9 , which was the center point. Treatment 9 was followed by treatment 2 , and then treatment 3 , at 8 and $6 \mathrm{~s}$, respectively. Treatment 6 was relatively similar to both treatment 3 and treatments 1 and 5. Treatments 1 and 5 were also relatively similar to treatment 3 , and treatment 3 was relatively similar to treatment 4 . Treatment 8 produced the lowest water stability with an average of $0.40 \mathrm{~s}$. According to Table 6, the moisture content of the raw ingredients, temperature of the die, and screw speed of the extruder did not result in significant relationships for the water stability values of the DDGS extrudates. Because DDGS contains very little starch, the pellets lack a binder typically provided by gelatinization of starch. According to Table 5, the increase in moisture content caused an increase in water stability of the corn extrudates, but corn contains large starch content. An increase in both the screw speed of the extruder and the temperature of the die caused a decrease in the water stability of the corn extrudates. It is clear that in order to be effectively used in aquafeeds, DDGS cannot be used alone; instead it must be processed with other ingredients which, hopefully, will improve particle binding characteristics.

\subsubsection{Floatability}

Floatability is the amount of time the pellets float on top of the water. It is important in aquaculture feeds because some species only eat the feed if it is floating, and the pellets must float for approximately 30 min for appropriate feeding time. According to Table 4, the greatest floatability was produced by treatments 2 and 4 at $3.33 \mathrm{~s}$, while the lowest floatability was produced by treatments 3 and 9 at $2 \mathrm{~s}$. Treatments 1, 5, 6, 7, and 8 were all relatively similar to one another. There was no direct relationship between the moisture content and screw speed and the floatability of the DDGS extrudates, according to Table 6. As the temperature of the extruder increased, the floatability of the DDGS extrudates increased, due to greater expansion. According to Table 5, as the moisture content of the raw corn blends increased, the floatability of the corn extrudates decreased. As the screw speed and temperature of the extruder increased, the floatability of the corn extrudates decreased (Table 5).

\subsubsection{Bulk Density}

The bulk density quantifies the amount of mass a specific volume can hold. Bulk density is important in storage of aquaculture feeds because the pellets will be distributed in containers, and the containers need to hold as much mass as possible. According to Table 4, the greatest bulk density was produced by treatment 3 at $399.00 \mathrm{~kg} / \mathrm{m}^{3}$. Treatments 7 and 8 followed treatment 3 , having slightly lower bulk density. Treatment 1 was relatively similar to treatments 7 and 8 , as well as treatment 2 . Treatment 5 had a bulk density of $355.00 \mathrm{~kg} / \mathrm{m}^{3}$, which was slightly lower than treatment 2 . The smallest value for bulk density was produced by treatment 6 , with a value of 324.73 $\mathrm{kg} / \mathrm{m}^{3}$. According to Table 6 , as the moisture content of the raw DDGS blend increased, the bulk density of the DDGS increased. The bulk density of the DDGS extrudates decreased as the temperature and screw speed of the extruder increased, due to greater expansion. According to Table 5, similar results were produced for the corn extrudates.

\subsection{Implications}

Figure 2 is a matrix plot of all independent and dependent variables, and it shows that the extruded DDGS had much different behavior than the extruded corn for all properties studied. This was true for all screw speeds, all ingredient moistures, and all extruder temperatures. This occurred due to the chemical composition being substantially different. As Liu and Rosentrater (2011) discussed, DDGS has approximately three times the levels of protein, fiber, fat, and minerals vis-à-vis corn, whereas DDGS has substantially lower starch (because alcohol manufacturing utilizes only the starch component from the corn kernels). Use of DDGS vs. corn in aquafeed formulations definitely impacts extrudate quality, as shown in Figure 2. 


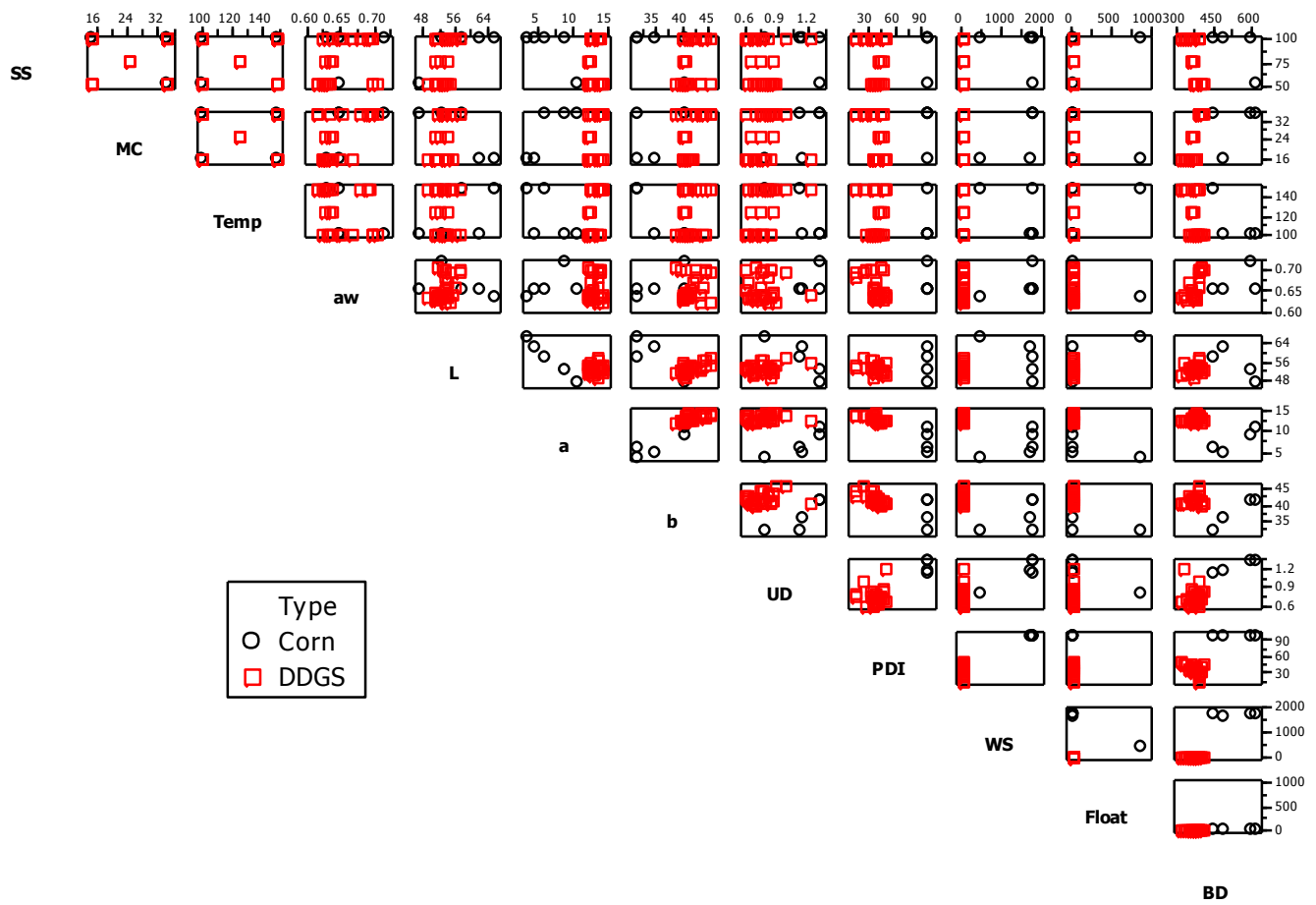

Figure 2. Matrix plot of all independent and dependent variables shows that the extruded DDGS had much different behavior than the extruded corn for all properties studied.

\section{Conclusion}

In conclusion, the moisture content of the raw ingredients led to an increase in the water activity in the raw ingredients. It also led to an increase in moisture content, water activity level, $\mathrm{L}^{*}$ values, $\mathrm{b}^{*}$ values, and bulk density of the DDGS extrudates. An increase in moisture content led to a decrease in pellet durability index. There was no significant relationship between the moisture content of the raw ingredients and the $\mathrm{a}^{*}$ values, unit density, water stability, or floatability of the DDGS extrudates. The increase in temperature of the extruder die led to an increase in the $a^{*}$ values, $b^{*}$ values, unit density, and floatability of the DDGS extrudates. An increase in the temperature of the die led to a decrease in the moisture content, water activity level, and bulk density of the DDGS extrudates. There was no significant relationship between the temperature of the die and the $\mathrm{L}^{*}$ values, pellet durability index, or water stability of the DDGS extrudates. The increase in screw speed caused an increase in the moisture content, water activity level, $\mathrm{L}^{*}$ values, and $\mathrm{b}^{*}$ values of the DDGS extrudates. An increase in the screw speed of the extruder caused a decrease in the pellet durability index and bulk density of the extrudates. There was no significant relationship between the screw speed of the extruder and the $a^{*}$ values, unit density, water stability, and floatability of the DDGS extrudates.

Ultimately, the composition of the ingredients affects the resulting physical properties of the extrudates. The amount of starch and water present in the ingredients determines the quality of the pellets. Because starch gelatinizes and acts as a binding agent, the more starch present, the better the quality of the pellet. Along with starch content, the amount of fiber and protein in the ingredients affects the quality of the extrudates. It is important to have a high protein diet for aquaculture feed, so the fish reach substantial weight in a reasonable amount of time. The key is to find an appropriate balance between moisture content, screw speed, and temperature, as well as the starch, protein, and fiber content of the ingredients. It is clear that DDGS cannot be processed alone at the extrusion conditions used in this study. DDGS should be used in combination with other ingredients in order to balance nutrient levels as well as improve feed quality characteristics.

\section{References}

AACC. (2000). Method 44-19, moisture-air oven method, drying at $135^{\circ} C$. AACC Approved Methods (10 ${ }^{\text {th }}$ ed.). St. Paul, MN: American Association of Cereal Chemists.

Ayadi, F. Y., Muthukumarappan, K., Rosentrater, K. A., \& Brown, M. L. (2011a). Single-screw extrusion processing of distillers dried grains with solubles (DDGS)-based yellow perch (Perca flavescens) feeds. 
Cereal Chemistry, 88(2), 179-188. http://dx.doi.org/10.1094/CCHEM-08-10-0118

Ayadi, F. Y., Muthukumarappan, K., Rosentrater, K. A., \& Brown, M. L. (2011b). Twin-screw extrusion processing of rainbow trout (Oncorhynchus mykiss) feeds using various levels of corn-based distillers dried grains with solubles (DDGS). Cereal Chemistry, 88(4), 363-374. http://dx.doi.org/10.1094/CCHEM-08-10-0120

Ayadi, F. Y., Rosentrater, K. A., Muthukumarappan, K., \& Brown M. L. (2011c). Twin-screw extrusion processing of distillers dried grains with solubles (DDGS)-based yellow perch (Perca flavescens) feeds. Food and Bioprocess Technology, 5(5), 1963-1978.

Chevanan, N., Muthukumarappan, K., \& Rosentrater, K. A. (2007a). Extrusion studies of aquaculture feed using distillers dried grains with solubles and whey. Food and Bioprocess Technology, 2, 177-185. http://link.springer.com/content/pdf/10.1007\%2Fs11947-007-0036-8.pdf

Chevanan, N., Muthukumarappan, K., Rosentrater, K. A., \& Julson, J. L. (2007b). Effect of die dimensions on extrusion processing parameters and properties of DDGS-based aquaculture feeds. Cereal Chemistry, 84(4), 389-398. http://dx.doi.org/10.1094/CCHEM-84-4-0389

Chevanan, N., Rosentrater, K. A., \& Muthukumarappan, K. (2007c). Twin-screw extrusion processing of feed blends containing distillers dried grains with solubles (DDGS). Cereal Chemistry, 84(5), 428-436. http://dx.doi.org/10.1094/CCHEM-84-5-0428

Chevanan, N., Rosentrater, K. A., \& Muthukumarappan, K. (2008a). Effect of DDGS, moisture content, and screw speed on physical properties of extrudates in single-screw extrusion. Cereal Chemistry, 85(2), 132-139. http://dx.doi.org/10.1094/CCHEM-85-2-0132

Chevanan, N., Rosentrater, K. A., \& Muthukumarappan, K. (2008b). Effects of processing conditions on single screw extrusion of feed ingredients containing DDGS. Food and Bioprocess Technology, 3(1), 111-120. http://link.springer.com/content/pdf/10.1007\%2Fs11947-008-0065-y.pdf

Delgado-Nieblas, C., Aguilar-Palazuelos, E., Gallegos-Infante, A., Rocho-Guzman, N., Zazueta-Morales, J., \& Caro-Corrales, J. (2012). Characterization and optimization of extrusion cooking for the manufacture of third-generation snacks with winter squash (Cucurbita moschata D.) flour. Cereal Chemistry, 89(1), 65-72. http://dx.doi.org/10.1094/CCHEM-02-11-0016

Forsido, S., \& Ramaswamy, H. S. (2011). Protein rich extruded products from tef, corn and soy protein isolate blends. Ethiopian Journal of Applied Sciences and Technology, 2(2), 75-90. http://www.researchgate.net/publication/224872856_Protein_Rich_Extruded_Products_from_Tef_Corn_an d_Soy_Protein_Isolate_Blends

Kannadhason, S., Muthukumarappan, K., \& Rosentrater, K. A. (2008). Effect of starch sources and protein content on extruded aquaculture feed containing DDGS. Food and Bioprocess Technology, 4, 282-294. http://link.springer.com/content/pdf/10.1007\%2Fs11947-008-0177-4.pdf

Kannadhason, S., Muthukumarappan, K., \& Rosentrater, K. A. (2009). Effects of ingredients and extrusion parameters on aquafeeds containing DDGS and tapioca starch. Journal of Aquaculture Feed Science and Nutrition, 1(1), 6-21. http://www.medwelljournals.com/fulltext/?doi=joafsnu.2009.6.21

Kannadhason, S., Rosentrater. A., Muthukumarappan, K., \& Brown, M. L. (2010). Twin-screw extrusion on DDGS-based aquaculture feeds. Journal of the World Aquaculture Society, 41(S1), 1-13. http://dx.doi.org/10.1111/j.1749-7345.2009.00328.x

Lardy, G. (2013). Feeding corn to beef cattle. Retrieved June 1, 2013, from http://www.ag.ndsu.edu/pubs/ansci/beef/as1238.pdf

Liu, K., \& Rosentrater, K. (2011). Distillers Grains: Production, Properties, and Utilization. Boca Raton, FL: CRC Press.

Singh, R. K., Majumdar, R. K., \& Venkateshwarlu, G. (2012). Optimum extrusion-cooking conditions for improving physical properties of fish-cereal based snacks by response surface methodology. Journal of Food Science and Technology. http://link.springer.com/content/pdf/10.1007\%2Fs13197-012-0725-9.pdf

Razzaq, M. R., Anjum, F. M., Khan, M. I., Khan, M. R., Nadeem, M., Javed, M. S., \& Sajid, M. W. (2012). Effect of temperature, screw speed and moisture variations on extrusion cooking behavior of maize (Zea mays. L). Pakistan Journal Food Sciences, 22(1), 12-22.

http://www.psfst.com/_jpd_fstr/c2fda8f7cfc6ffb79f9d45ab1cd36929.pdf 
Rosentrater, K. A., Muthukumarappan, K., \& Kannadhason, S. (2009a). Effects of ingredients and extrusion parameters on properties of aquafeeds containing DDGS and corn starch. Journal of Aquaculture Feed Science and Nutrition, 1(2), 44-60. http://www.medwelljournals.com/fulltext/?doi=joafsnu.2009.44.60

Rosentrater, K. A., Muthukumarappan, K., \& Kannadhason, S. (2009b). Effects of ingredients and extrusion parameters on aquafeeds containing DDGS and potato starch. Journal of Aquaculture Feed Science and Nutrition, l(1), 22-38.http://docsdrive.com/pdfs/medwelljournals/joafsnu/2009/22-38.pdf

Simons, C. W., Hall, C., \& Tulbek, M. (2012). Effects of extruder screw speeds on physical properties and in vitro starch hydrolysis of precooked pinto, navy, red, and black bean extrudates. Cereal Chemistry, 89(3), 176-181. http://dx.doi.org/10.1094/CCHEM-08-11-0104

Sorensen, M. (2012). A review of the effects of ingredient composition and processing conditions on the physical qualities of extruded high-energy fish feed as measured by prevailing methods. Aquaculture Nutrition, 18, 233-248. http://dx.doi.org/10.1111/j.1365-2095.2011.00924.x

USB. (2012). Soy Nutritional Content. Soy Connection. Retrieved June, 1, 2013, from http://www.soyconnection.com/health_nutrition/technical_info/protein_content.php.

USDA. (1999). Practical procedures for grain handlers: inspecting grain. Grain Inspection, Packers, and Stockyards Administration, Washington DC, USA. Retrieved June, 1, 2013, from http://archive.gipsa.usda.gov/pubs/primer.pdf.

\section{Copyrights}

Copyright for this article is retained by the author(s), with first publication rights granted to the journal.

This is an open-access article distributed under the terms and conditions of the Creative Commons Attribution license (http://creativecommons.org/licenses/by/4.0/). 